Document downloaded from:

http://hdl.handle.net/10251/102286

This paper must be cited as:

Lucas Borja, A.; Belenguer, A.; Esteban González, H.; Boria Esbert, VE. (2017). Design and Performance of a High-Q Narrow Bandwidth Bandpass Filter in Empty Substrate Integrated Coaxial Line at Ku-Band. IEEE Microwave and Wireless Components Letters. 27(11):977979. doi:10.1109/LMWC.2017.2750118

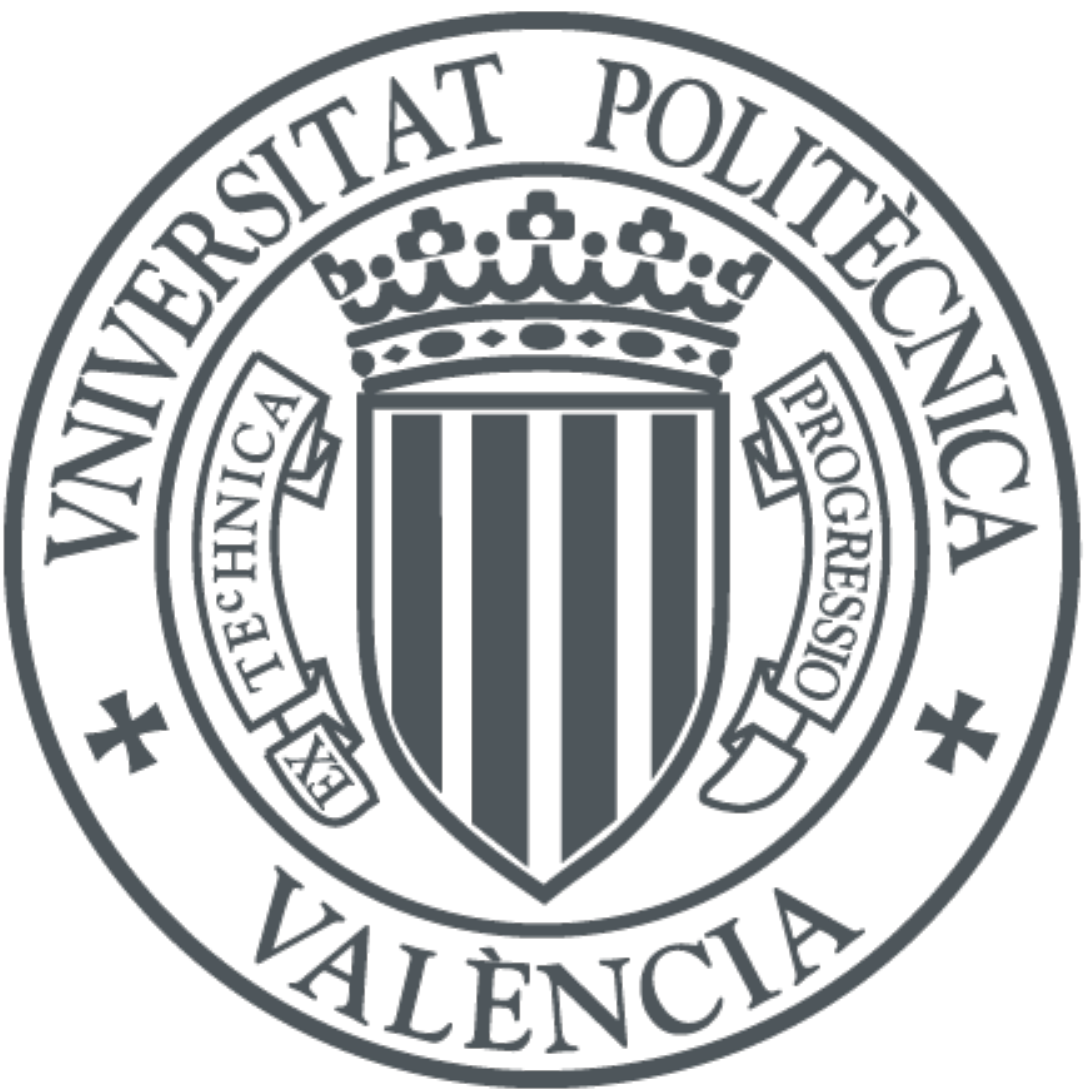

The final publication is available at

http://doi.org/10.1109/LMWC.2017.2750118

Copyright Institute of Electrical and Electronics Engineers

Additional Information 


\title{
Design and Performance of a High-Q Narrow Bandwidth Bandpass Filter in Empty Substrate Integrated Coaxial Line at $K_{u}$-Band
}

\author{
Alejandro L. Borja, Member, IEEE, Angel Belenguer, Senior Member, IEEE, \\ Hector Esteban, Senior Member, IEEE, and Vicente E. Boria, Senior Member, IEEE
}

\begin{abstract}
This paper presents the design and performance of a planar narrow bandwidth bandpass filter with high quality factor. The structure is composed of an empty substrate integrated coaxial line with the center conductor suspended in air. The component dimensions have been calculated by means of classical microwave filter design theory. The filter has been manufactured using standard printed circuit board fabrication processes. A measured insertion loss of $1.59 \mathrm{~dB}, 2.93 \%$ fractional bandwidth and Q-factor of 1505 have been obtained at 15 GHz. A Qfactor comparison with other substrate and empty substrate integrated technologies shows the advantages of the solution considered in this work. The proposed filter proves to be suitable for the implementation of integrated microwave or millimeterwave subsystems with severe restrictions, i.e. low in-band losses, very narrow bandpass responses, low frequency dispersion, high out-of-band rejection and low manufacturing cost.
\end{abstract}

Index Terms-Empty substrate integrated coaxial line (ESICL), Empty substrate integrated waveguide (ESIW), Substrate integrated waveguide (SIW)

\section{INTRODUCTION}

During the last years, great efforts have been devoted to the development of novel structures that can be integrated within planar circuits. In this regard, the synthesis of the first substrate integrated waveguide (SIW) [1] using a standard printed circuit board (PCB) technique has produced a huge progress on this field. Complete metallized walls, or linear periodic arrays of metal via-holes, within a substrate layer were used to enclose the wave-propagation area. The proposed methodology has allowed to incorporate non-planar structures to common (microwave or millimeter-wave) planar circuits with low cost. Unfortunately, its use is limited at high frequencies due to substrate losses, and consequent reduction of the quality factor $(\mathrm{Q})$ in filtering applications. To overcome these problems, new substrate-free arrangements have been studied due their reduced losses. For instance, novel empty substrate waveguides were recently proposed in [2]. Concerning coaxial lines, air-filled or partially air-filled structures based on stacked

This work was supported by the Ministerio de Ciencia e Innovación, Spanish Goverment, under Research Projects TEC2016-75934-C4-X-R and TEC2016-75934-C4-1-R.

A. L. Borja and A. Belenguer are with Departamento de Ingeniería eléctrica, electrónica, automática y comunicaciones, Escuela Politécnica de Albacete, Campus Universitario, 02071 Albacete and Escuela Politécnica de Cuenca, Campus Universitario, 16071 Cuenca, Universidad de Castilla-La Mancha, Spain (e-mail: alejandro.lucas@uclm.es; angel.belenguer@uclm.es;).

H. Esteban and V. E. Boria are with Departamento de Comunicaciones, Universidad Politécnica de Valencia, 46022 Valencia, Spain (e-mail: hesteban@dcom.upv.es; vboria@dcom.upv.es;). metallic layers have been studied [3] - [5]. Nevertheless, these structures are not fully integrated, usually involving complex and expensive fabrication procedures, and they do not present transitions from/to other planar transmission lines. In order to further explore the advantages of empty substrate transmission lines, we present in this paper a planar coaxial filter with low losses and high Q-factor for operation at $K_{u}$-band. Due to its non-dispersive behaviour, coaxial transmission lines are commonly employed for wideband filtering applications [6]. However, the advantages of these coaxial filters for narrow or very narrow passband operation are analyzed in this work.

\section{ESICL BANDPASS FILTER DESIGN}

The narrow bandwidth bandpass filter based on empty substrate integrated coaxial lines (ESICLs) has been designed by means of the well-known insertion loss method (ILM) fully described in [7]. First, the lowpass filter prototype is designed considering normalized values for the source impedance and cutoff frequency, and then a frequency transformation is applied in order to obtain the requested bandpass filter specifications. In this work, a Tchebyscheff bandpass response with $3.3 \%$ fractional bandwidth at $15 \mathrm{GHz}, 4$ poles and 0.02 $\mathrm{dB}$ ripple along the passband has been designed. The ideal prototype and its characteristic values calculated through the aforementioned design procedure are shown in Fig. 1. A bandpass filter composed of five impedance inverters and four series resonators is firstly obtained.

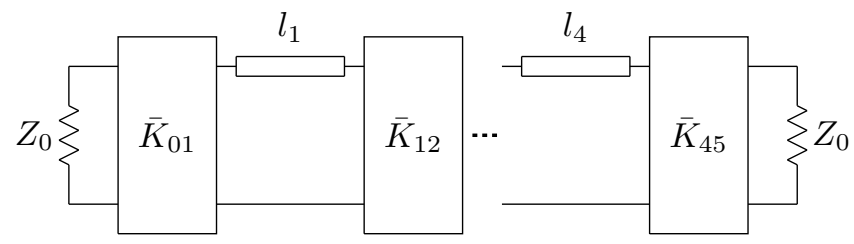

Fig. 1. Bandpass filter based on series resonators and impedance inverters. Ideal filter parameters are $l_{1}=l_{4}=9 \mathrm{~mm}, l_{2}=l_{3}=9.7 \mathrm{~mm}, \bar{K}_{01}=$ $\bar{K}_{45}=0.2571, \bar{K}_{12}=\bar{K}_{34}=0.0509, \bar{K}_{23}=0.0374$ and $Z_{0}=50 \Omega$.

Once the bandpass prototype has been determined, it is implemented by means of series resonators (using short-circuited $\lambda / 2$ ESICL transmission lines) and impedance inverters (using inductances connected to ground) for achieving its practical coupling. The equivalent implementation using ESICL lines can be accomplished with the structure proposed in Fig. 2 . 
The equations required to obtain the negative phase and shunt inductance of the inverters are as follows:

$$
\begin{gathered}
\bar{\phi}_{n, m}=\frac{1}{2} \operatorname{atan}\left(2 \bar{X}_{n, m}\right), \bar{X}_{n, m}=\frac{\bar{K}_{n, m}}{1-\bar{K}_{n, m}^{2}}, n=1, \ldots, N \\
\bar{L}_{n}=\frac{\bar{X}_{n-1, n}}{\omega_{0}}, n=1, \ldots, N+1
\end{gathered}
$$

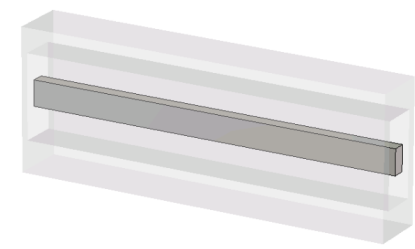

(a) ESICL $\lambda / 2$ transmission line

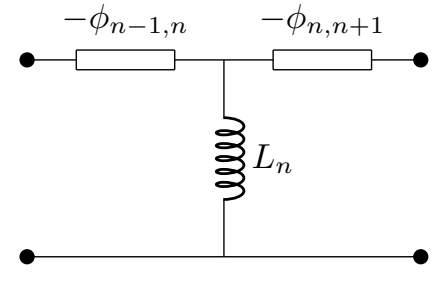

(b) Lumped element circuit of the impedance inverter

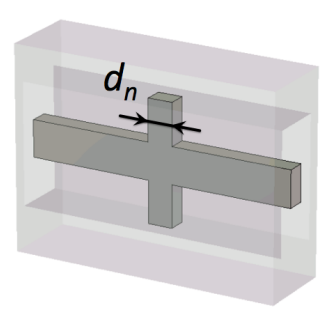

(c) ESICL impedance inverter
Fig. 2. Planar ESICL transmission line. Dark and light grey represent the inner and outer conductor of the coaxial line, respectively.

Next, the dimensions of each impedance inverter are designed and optimized. This goal has been obtained by matching the full-wave simulated scattering parameters of the lossless inverter (see Fig. 2(c)) and its associated lumped circuit response (see Fig. 2(b)). Fig. 3 shows the simulated matched response for the first and last inverter after optimization.

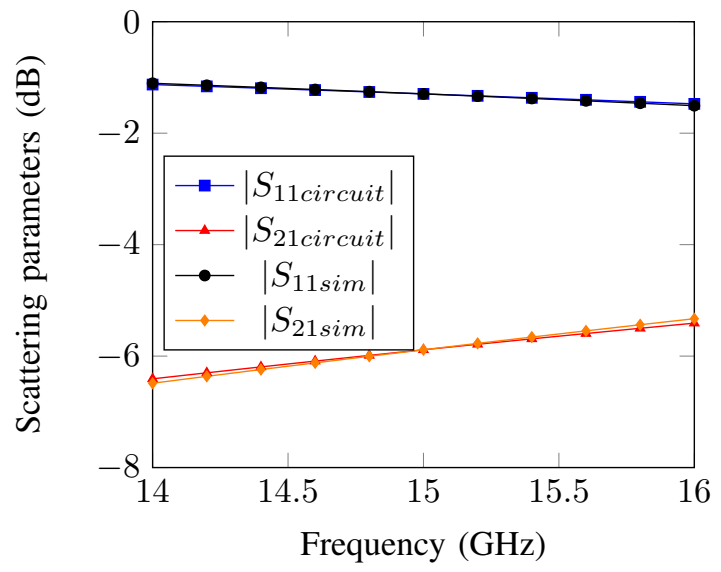

Fig. 3. Simulated lumped element circuit $\left(\left|S_{11 \text { circuit }}\right|\right.$ and $\left.\left|S_{21 \text { circuit }}\right|\right)$ and full-wave $\left(\left|S_{11 \text { sim }}\right|\right.$ and $\left.\left|S_{21 \text { sim }}\right|\right)$ scattering parameters of the inverter.

Finally, the resonant transmission lines and the impedance inverters are connected. It is worth mentioning that, at this point, the negative phases from previous and later inverters are removed from the corresponding $\lambda / 2$ transmission lines resonators. In addition, two feeding lines at the input and output ports are added to the structure. The final and real arrangement of the passband filter is shown in Fig. 4.

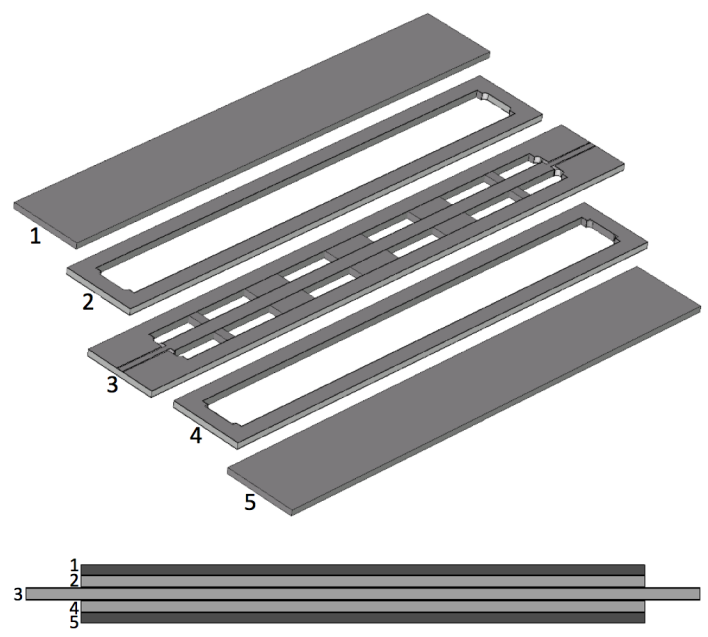

(a) Layers and side view

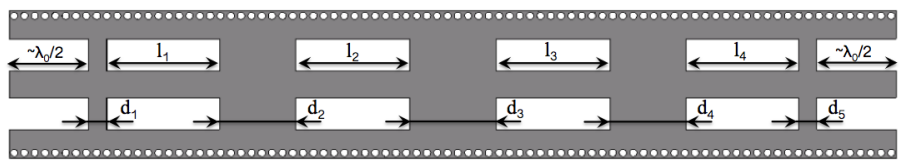

(b) Central layer geometry

Fig. 4. Layouts of the proposed empty substrate integrated coaxial line. $\lambda_{0}=19,99, w_{c e}=6, w_{c i}=1.9173, l_{1}=l_{4}=10.7913, l_{2}=l_{3}=$ $13.1878, d_{1}=d_{5}=1.2302, d_{2}=d_{4}=5.1308$ and $d_{3}=5.8234$. All dimensions are in $\mathrm{mm}$.

The structure is composed of different substrate layers and metallic covers numbered from 1 to 5 . Layers 1 and 5 are cover sheets used to confine the electromagnetic waves inside the transmission line. Layers 2 and 4 are included to separate the covers from the central line named layer 3 . This central layer contains the series resonators and impedance inverters. A Rogers $4003 \mathrm{C}$ substrate with permittivity $\epsilon_{r}=3.55$, thickness $h=0.813 \mathrm{~mm}$ and loss tangent $\tan \delta=0.0025$ has been chosen for layers 2, 3 and 4, while an FR4 substrate has been utilized for covers 1 and 5 . It is worth noting that the substrate properties just affect to the CPW feeding lines, as the rest of the structure is completely metallized. Thus, very cheap dielectric substrate layers could be employed in all layers without involving a high degradation of the filter performance. Simulated results predict an increment of 0.8 $\mathrm{dB}$ if FR4 substrate was used to fabricate all layers, namely layers 1 to 5 .

The excellent response obtained in terms of insertion losses and selectivity for this narrow-band filter is related to the high $\mathrm{Q}$ of the proposed technology. For comparison, the unloaded Q $\left(Q_{u}\right)$ of the ESICL, and of other well known transmission lines, have been calculated for different simulated $\lambda / 2$ resonators weakly coupled to input/output ports. The results show that the ESICL line presents the second highest $Q_{u}$ value for both calculations, i.e. 1567 using [8] and 1566 for CST, only below the classical rectangular waveguide (RWG) technology 
with a $Q_{u}$ of 6134 for [8] and 6883 with CST. To the best of the authors' knowledge, the $Q_{u}$ obtained for the ESICL is one of the highest values achieved for planar resonators. The performance of the empty coaxial line is similar to the behavior of the empty substrate integrated waveguide (ESIW) line [2], that has a slightly lower $Q_{u}$ of around 1487 using [8] and 1339 for CST. In addition, the ESICL has lower dispersion compared to the ESIW. As it can be expected, the SIW and microstrip lines have significantly lower $Q_{u}$, below 300 and 24 , respectively, due to the presence of dielectric layers that increase the total losses of these structures.

\section{Fabricated Prototype And Measured Results}

The manufactured prototype of the ESICL narrow bandwidth bandpass filter is shown in Fig. 5. Since the implementation of the empty integrated coaxial filter follows standard processes (drilling, milling, and metallizing), it can be fabricated with low cost, easy assembling and mass-production techniques.

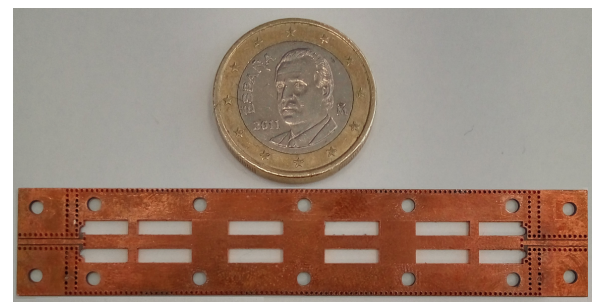

(a) Fabricated central layer

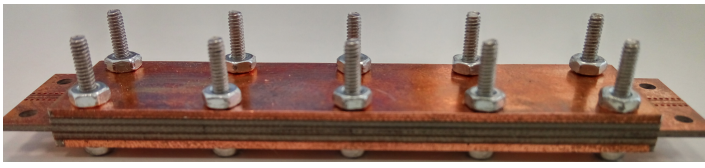

(b) Assembled structure

Fig. 5. Fabricated empty substrate integrated coaxial filter.

Fig. 6 illustrates the simulated and measured frequency responses of the filter. Experimental results agree well with numerical data. The structure exhibits a measured narrow passband from $14.8 \mathrm{GHz}$ to $15.24 \mathrm{GHz}(2,93 \% \mathrm{FBW})$ with high frequency selectivity at both band edges. The measured out-of-band rejection is better than $-40 \mathrm{~dB}$ at $1 \mathrm{GHz}$ from the central frequency. Furthermore, the filter presents a flat response with low ripple, and the reflection coefficient is below $-23 \mathrm{~dB}$ along the passband. The measured insertion losses at the filter central frequency are $1.59 \mathrm{~dB}$, whereas the simulated losses are $1.08 \mathrm{~dB}$. The simulations and measurements include $\mathrm{K}$ connectors and taper transitions.

In addition, the unloaded quality factor of the measured filter has been calculated using the formulation described in [9]. The final $Q_{u}$ achieved for the measured filter without transitions and $\mathrm{K}$-connectors is 1505 , which is quite close to the value of 1566 provided by CST. The differences between both results can be attributed to fabrication tolerances and imperfections due to PCB roughness and non-ideal plated metals.

The proposed coaxial filter could be employed in applications where high selectivity, negligible frequency dispersion, low insertion losses, and low ripple levels are required.

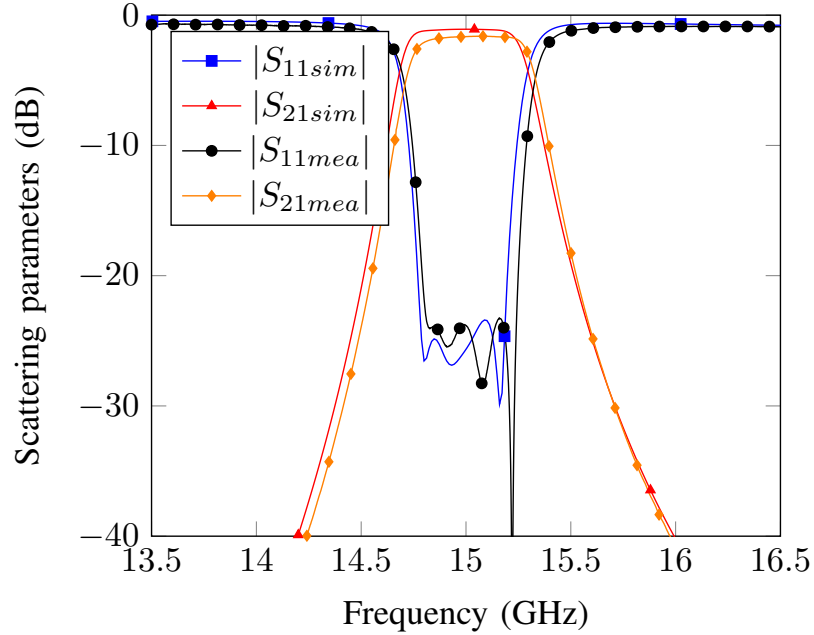

Fig. 6. Final simulated and measured scattering parameters.

\section{CONClusions}

A novel empty substrate integrated coaxial narrow bandwidth bandpass filter with high $\mathrm{Q}$ for operation at $K_{u}$ band has been designed, fabricated, and measured. Particularly, the fabricated prototype exhibits an insertion loss value of 1.59 $\mathrm{dB}$ with a $2.93 \%$ fractional bandwidth at $15 \mathrm{GHz}$. This low insertion loss value is due to the high unloaded $Q$ that can be obtained with ESICL transmission lines. The measured $Q_{u}$ value obtained is 1505 , which is one of the highest values achieved for planar filters operating in this high frequency range.

\section{REFERENCES}

[1] D. Deslandes and K. Wu, "Integrated microstrip and rectangular waveguide in planar form," Microwave and Wireless Components Letters, IEEE, vol. 11, no. 2, pp. 68-70, Feb 2001.

[2] A. Belenguer, H. Esteban, and V. Boria, "Novel empty substrate integrated waveguide for high-performance microwave integrated circuits," Microwave Theory and Techniques, IEEE Transactions on, vol. 62, no. 4, pp. 832-839, April 2014.

[3] I. Llamas-Garro, M. J. Lancaster, and P. S. Hall, "Air-filled square coaxial transmission line and its use in microwave filters," IEE Proceedings Microwaves, Antennas and Propagation, vol. 152, no. 3, pp. 155-159, June 2005.

[4] M. J. Lancaster, J. Zhou, M. Ke, Y. Wang, and K. Jiang, "Design and high performance of a micromachined K-band rectangular coaxial cable," IEEE Transactions on Microwave Theory and Techniques, vol. 55, no. 7, pp. 1548-1553, July 2007.

[5] N. Jastram and D. Filipovic, "PCB-based prototyping of 3-D micromachined RF subsystems," Antennas and Propagation, IEEE Transactions on, vol. 62, no. 1, pp. 420-429, Jan 2014.

[6] A. Belenguer, A. L. Borja, H. Esteban, and V. Boria, "High-performance coplanar waveguide to empty substrate integrated coaxial line transition," Microwave Theory and Techniques, IEEE Transactions on, vol. 63, no. 12, pp. 4027-4034, Dec 2015.

[7] G. L. Matthaei, L. Young, and E. M. T. Jones, Chapter 8 "Band-pass filters (a general summary of band-pass filters and a versatile design technique for filters with narrow or moderate bandwidths)" in. Artech House, Inc., 1985.

[8] X.-C. Zhu, W. Hong, K. Wu, K.-D. Wang, L.-S. Li, Z.-C. Hao, H.-J. Tang, and J.-X. Chen, "Accurate characterization of attenuation constants of substrate integrated waveguide using resonator method," Microwave and Wireless Components Letters, IEEE, vol. 23, no. 12, pp. 677-679, Dec 2013.

[9] J.-S. Hong and M. Lancaster, Microstrip filters for RF/microwave applications. John Wiley \& Sons, Inc., 2001. 